\title{
Potential Impact of Nirsevimab on RSV Transmission and Medically Attended Lower Respiratory Tract Illness Caused by RSV: A Disease Transmission Model
}

\author{
Nicolas Voirin $\cdot$ Victor Virlogeux $\cdot$ Clarisse Demont $\cdot$ Alexia Kieffer
}

Received: August 23, 2021 / Accepted: November 9, 2021 / Published online: November 23, 2021

(C) The Author(s) 2021

\begin{abstract}
Introduction: Respiratory syncytial virus (RSV) is associated with significant morbidity worldwide, especially among infants. We evaluated the potential impact of prophylactic nirsevimab, a monoclonal antibody, in infants experiencing their first RSV season, and the number of medically-attended lower respiratory tract infection episodes caused by RSV (RSVMALRTI) in the USA.

Methods: We developed an age-structured, dynamic, deterministic compartmental model reflecting RSV natural history, incorporating USA demographic data and an age-specific contact matrix. We assumed either no effect of
\end{abstract}

Supplementary Information The online version contains supplementary material available at https:// doi.org/10.1007/s40121-021-00566-9.

N. Voirin · V. Virlogeux

Epidemiology and Modelling in Infectious Diseases

(EPIMOD), Lent, France

V. Virlogeux

Hospices Civils de Lyon, Lyon, France

C. Demont

Vaccine and Epidemiology Modelling, Sanofi

Pasteur, Lyon, France

A. Kieffer $(\square)$

Health Economics and Value Assessment, Sanofi

Pasteur, Siège Mondial, 14 Espace Henry Vallée,

69007 Lyon, France

e-mail: alexia.kieffer@sanofi.com nirsevimab on transmission (scenario 1) or a $50 \%$ reduction of viral shedding (scenario 2 ). Model outcomes were RSV-MALRTIs, ICD-9 coded in the Marketscan ${ }^{\circledR}$ database by month. We focused on age groups corresponding to the first 2 years of life, during seven RSV seasons (2008-2015).

Results: Scenario 1 illustrated the direct individual benefit when a universal immunization strategy is applied to all infants. In scenario 2, herd protection was observed across age groups, with $15.5 \%$ of all avoided cases due to reduced transmission; the greatest impact was in the youngest age group and a benefit was observed in those aged $65+$ years.

Conclusion: These preliminary data suggest that single-dose nirsevimab will benefit infants experiencing their first RSV season, with a potential increase in effectiveness dependent on nirsevimab's mechanism of action.

Keywords: Disease transmission model; Immunization/prophylaxis strategy; Medically attended lower respiratory tract illness; Nirsevimab; RSV 


\section{Key Summary Points}

Nirsevimab is a monoclonal antibody developed as a passive immunization strategy to prevent lower respiratory tract infection (LRTI) caused by respiratory syncytial virus (RSV) in all infants experiencing their first RSV season

Dynamic mathematical models can provide initial insights into the direct and indirect effects of nirsevimab on RSV transmission, taking into account the uncertainties surrounding its mechanism of action

We used a deterministic, age-structured, compartmental RSV transmission model to assess the potential impact of nirsevimab on the prevention of medically attended LRTI caused by RSV in the overall population in the USA, with an unprecedented level of granularity in age groups $<2$ years

The model showed significant benefit of prophylactic nirsevimab on RSV when administered to all infants during their first season; this benefit increased upon assumption of an effect of nirsevimab on viral shedding

These preliminary data suggest that singledose nirsevimab may provide considerable benefit to infants experiencing their first RSV season

\section{INTRODUCTION}

Respiratory syncytial virus (RSV) is a leading cause of lower respiratory tract illness (LRTI) in infants and young children, with an estimated 33.1 million annual cases of RSV-associated LRTI in children $<5$ years old worldwide [1]. RSV is seasonal, with the timing of epidemics varying by region. In Europe and the USA, RSV disease burden generally occurs from November to March [2, 3].

Approximately $70 \%$ of infants $\leq 1$ year old become infected by RSV, and almost all children $\leq 2$ years old become infected at least once [4]. RSV-related healthcare utilization is highest for infants aged $<1$ year in the USA $[5,6]$; hospitalization rates due to RSV are 17 times higher than for flu in this age group [7]. Preterm infants and infants with underlying conditions such as chronic lung disease, cardiovascular disease, immunosuppression or neuromuscular disorders are at high risk of severe RSV episodes [8]. However, the disease mostly affects healthy infants, with $76 \%$ of RSV hospitalized infants being otherwise healthy and born at term [9].

With no specific treatment available, current therapeutic strategies aim to provide supportive care. The only approved prophylactic agent against RSV, palivizumab, is recommended in the USA for infants at high risk of significant morbidity or mortality [10]. This represents a small fraction of the USA birth cohort $(<3 \%$ of infants born each year within the Medicaid and commercially-insured populations) [11]. Among prophylactic approaches under development [12], nirsevimab is the most advanced. Nirsevimab is a monoclonal antibody developed as a passive immunization strategy to prevent LRTI caused by RSV in all infants experiencing their first RSV season [13]. Available data support the safety and efficacy of a single intramuscular dose of nirsevimab in preventing medically-attended LRTI (MALRTI) and hospitalizations for healthy preterm infants [14]; the evaluation of nirsevimab in healthy late preterm and term infants is ongoing (clinicaltrial.gov, NCT03979313) [15, 16].

The potential impact of nirsevimab on RSV circulation among infants requires examination. While effectiveness and real-life data will emerge in the years following potential licensure, dynamic mathematical models can provide initial insights into direct and indirect effects on RSV transmission. Monoclonal antibody RSV strategies have been previously evaluated using static modeling approaches $[17,18]$. Here, we used a deterministic, age-structured, compartmental RSV transmission model to assess the potential impact of nirsevimab on the 
prevention of MALRTI caused by RSV in the overall population in the USA, with an unprecedented level of granularity in age groups $<2$ years.

\section{METHODS}

\section{Mathematical Model}

We implemented a deterministic age-structured compartmental model reflecting RSV natural history, with similar structure as described elsewhere $[19,20]$. We focused on the first 2 years of life by including 1-month age intervals between birth and 24 months of age. We additionally included the age groups, 2-4, 5-9, $10-19, \quad 20-39, \quad 40-59, \quad 60-64, \quad 65-74$, and $\geq 75$ years.

The mathematical model is presented in Supplementary Material (Supplementary Methods; Supplementary Figures S1 and S2). Model parameters are presented in Table 1.

Table 1 Model parameters

\begin{tabular}{|c|c|c|c|c|}
\hline Parameters & Symbol & Average value & Range & References \\
\hline $\begin{array}{l}\text { Duration of maternal antibody } \\
\text { protection }\end{array}$ & $1 / \omega$ & 2 months & $1-3$ months & {$[21,22]$} \\
\hline $\begin{array}{l}\text { Duration of infectious period of } \\
1 \text { st, } 2 \text { nd and subsequent } \\
\text { infections }\end{array}$ & $1 / \gamma_{1,2,3}$ & $10,7,5$ days & - & {$[23,24]$} \\
\hline $\begin{array}{l}\text { Duration of short-term } \\
\text { immunity of recovered } \\
\text { individuals }\end{array}$ & $1 / p$ & 18.7 months (half-life: 7.4 months) & Estimated & {$[25,26]$} \\
\hline $\begin{array}{l}\text { Reduction of infectiousness for } \\
\text { 2nd and subsequent } \\
\text { infections }\end{array}$ & $\rho_{1,2}$ & $75 \%, 51 \%$ & - & {$[4,24,27-30]$} \\
\hline $\begin{array}{l}\text { Reduction of susceptibility after } \\
\text { 1st, 2nd, and subsequent } \\
\text { infections }\end{array}$ & $\sigma_{1,2}$ & $76 \%, 60 \%, 40 \%$ & - & {$[4,24,27-30]$} \\
\hline $\begin{array}{l}\text { Proportion of infections leading } \\
\text { to lower respiratory tract } \\
\text { infection by age groups }\end{array}$ & $d_{1,2,3}$ & $\begin{array}{l}d_{1,0-6 \text { months }}: 0.5, d_{1,7-12 \text { months }}: 0.4, d_{1}, \\
13-18 \text { months }: 0.3, d_{1,19-24 \text { months }}: 0.2, d_{1,} \\
2-4 \text { years: } 0.15, d_{1,5-9 \text { years }}: 0.15, d_{1,} \\
10-59 \text { years }: 0.1, d_{1,60-64 \text { years }}: 0.15, d_{1,} \\
65-74 \text { years }: 0.25, d_{1,75+\text { years }}: 0.4 \\
d_{2}=0.5 \times d_{1} \\
d_{3}=0.25 \times d_{1}\end{array}$ & - & $\begin{array}{l}\text { Adapted from } \\
\quad[4,26,28,31]\end{array}$ \\
\hline Seasonality parameters & $b, \varphi$ & $0.141,-2.831$ & Estimated & \\
\hline $\begin{array}{l}\text { Per contact transmission } \\
\text { probability }\end{array}$ & $\beta 0$ & 0.0054 & Estimated & \\
\hline Healthcare system use & $b$ & 0.396 & Estimated & \\
\hline
\end{tabular}




\section{Assumptions Related to the Impact of Nirsevimab}

The following assumptions were based on the target product profile of nirsevimab, confirmed by phase 2 clinical trial results [14]. Expected phase 3 results will allow further refinement of the set of assumptions related to the profile of nirsevimab. Single-dose nirsevimab was assumed to be administered to all infants with immediate and season-long (5 months) protection against severe RSV-LRTI, with a constant efficacy of $70 \%$ over the window of protection. We modeled a universal immunization strategy whereby nirsevimab is given to all infants aged 0-7 months entering their first RSV epidemic season or born during the epidemic season (1 November-31 March; Fig. 1). This strategy allows for protection of all infants experiencing their first RSV season, when the force of infection is higher, and through 12 months of age, when the risk of severe disease is higher. We assumed $71 \%$ coverage, as used in a recent impact model published by the USA Centers for Disease Control and Prevention [32].

Nirsevimab provides protection through passive immunization, by reducing viral load in the lower respiratory tract, thus preventing LRTI. Recent unpublished data (5th ReSViNET conference 2019, RSVVW'19) [33] shows that nirsevimab does not provide sterilizing immunity, thus the susceptibility of children to subsequent RSV infection will not be affected. However, it is unclear whether nirsevimab also reduces viral load in the upper respiratory tract of people, thereby affecting viral shedding from the host and subsequent transmission. Nirsevimab reduced RSV viral load in the upper and lower airways of cotton rats through its ability to block the $\mathrm{F}$ protein-mediated membrane fusion step in the viral entry process, neutralizing the virus [34]. We ran our model according to two scenarios: in scenario 1, we assumed no impact of nirsevimab on RSV transmission, while in scenario 2, we assumed RSV viral shedding can be reduced and assumed an arbitrary $50 \%$ reduction in transmission. The results from these two scenarios were compared with results obtained from no intervention, and the impact of nirsevimab was calculated based on the absolute reduction in the occurrence of RSV-MALRTI, by age group and season. Lexis diagrams were generated to visualize the impact

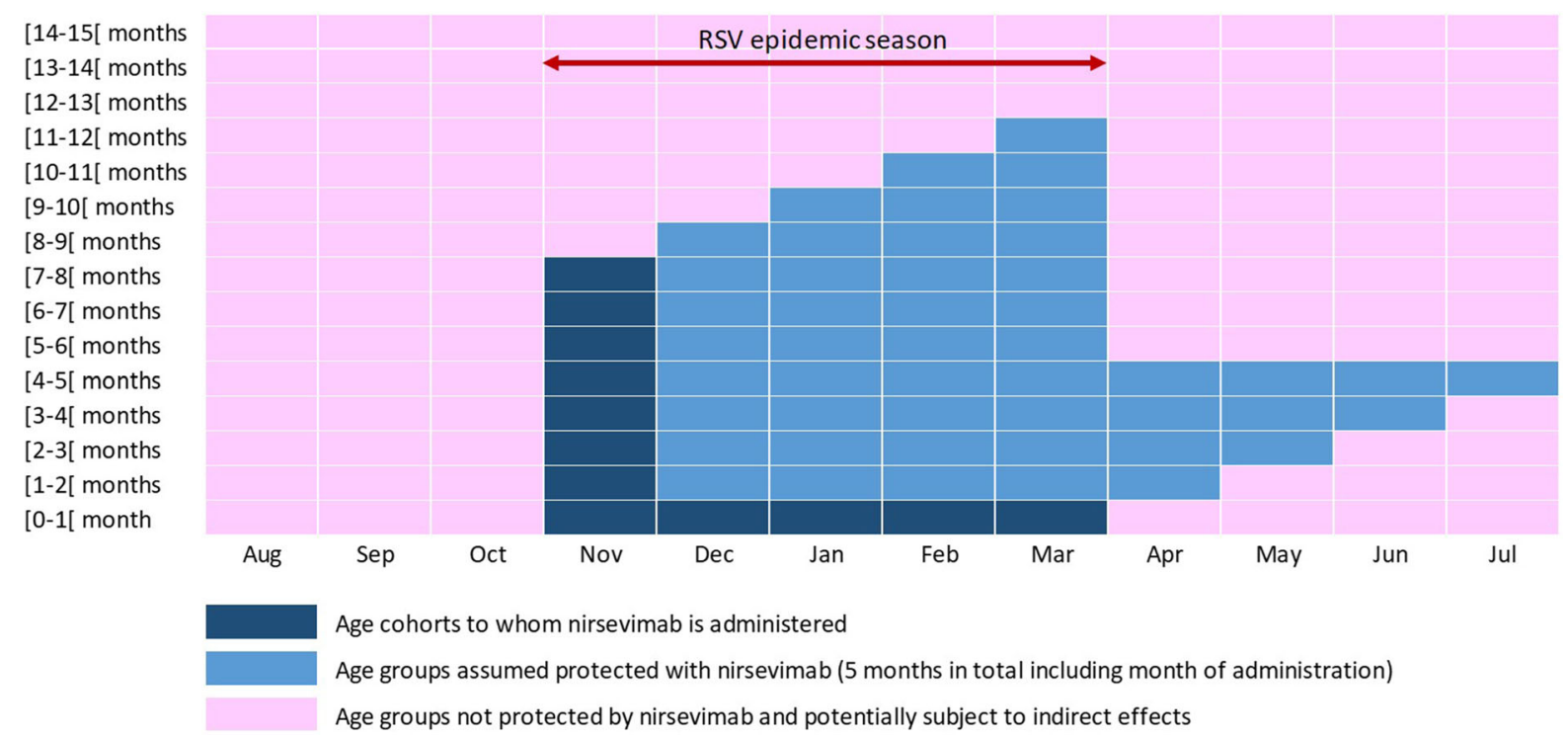

Fig. 1 Universal immunization schedule and duration of nirsevimab protection among infants aged 0-12 months during each season. Each age cohort can be followed diagonally from the dark blue boxes, representing the month when nirsevimab is administered, followed by four subsequent months of protection (light blue) 
of nirsevimab by age and by calendar month, spanning six seasons $(2009 / 2010$ to $2014 / 2015)$.

\section{Sensitivity Analyses}

Univariate and multivariate probabilistic sensitivity analyses assessed the potential impact of the following parameters on the natural history of the disease: the duration of maternal protective immunity between 1 and 3 months, the duration of short-term protection between 6 and 24 months, and the transmission parameter between $\beta_{0} / 2$ and $1.5 \times \beta_{0}$ in the model with no intervention. Additionally, for the models with interventions (scenarios 1 and 2), univariate probabilistic sensitivity analyses were performed for a coverage rate of nirsevimab varying between 0 and $100 \%$, and for a potential reduction in transmission varying from 0 to $100 \%$. We also conducted a sensitivity analysis for a possible overdiagnosis of RSV during RSV inter-epidemic periods and the contact matrix (see Supplementary Material; Supplementary Methods).

\section{Data Source and Definitions}

RSV-MALRTIs reported between 1 January 2008 and 31 December 2015 were obtained from the Truven Health Marketscan ${ }^{\circledR}$ Commercial Claims and Encounters, and Medicare Supplemental and Coordination of Benefits databases (Truven Health Analytics, MI, USA), which include longitudinal records for inpatient and outpatient services [35]. All database records were de-identified in compliance with US patient confidentiality requirements, including the Health Insurance Portability and Accountability Act of 1996. Institutional Review Board approval was not required as we used only deidentified patient records (Supplementary Material; Supplementary Methods).

\section{RESULTS}

\section{RSV-MALRTI Burden}

A seasonal pattern of RSV-MALRTIs from late fall to early spring was observed, overall and by age group (Supplementary Material, Figs. S3A and S3B). There were between 547,222 and 690,520 RSV-MALRTIs per season across the whole population (Supplementary Material, Table S1 and Figure S4). The highest burden was in infants aged 0-6 months and 6-12 months, with a seasonal average of 158,435 (25.1\%) and 141,237 (22.4\%) RSV-MALRTIs, respectively, and corresponding incidence rates of 71.6 and 71.1 per 1000 infants (Supplementary Material, Table S2). For age groups 12-18 months, 18-24 months, 2-4 years, 5-65 years, and $>65$ years, the incidence rates were 38.4 , $25.7,7.3,0.3$, and 0.7 per 1,000 persons, representing $13.1 \%, 8.1 \%, 14.0 \%, 12.8 \%$, and $4.4 \%$ of the overall seasonal burden, respectively.

\section{Model Calibration}

The model provided an accurate picture of RSVMALRTI seasonality, in terms of amplitude and timing of peak incidence during seasons (Fig. 2; Supplementary Material, Figures S5 and S6). With no intervention, the occurrence of RSVMALRTIs modeled over time, by age group up to 24 months, is shown in Fig. 3A.

\section{The Impact of Nirsevimab}

\section{Scenario 1: Assuming No Effect on Viral Shedding or RSV Transmission}

Assuming the immunization of the entire birth cohort and considering 71\% coverage rate, the monthly number of RSV-MALRTIs prevented with nirsevimab is presented by age group in Fig. 3B. The average reduction of RSV-MALRTIs during the RSV epidemic seasons was $49.7 \%$ $(\sim 58,000 \quad$ MALRTIs) among infants 
aged $<6$ months and 34.9\% $(\sim 38,000$ MALRTIs) among infants aged 6-12 months (Table 2A).

Consistent with the seasonal schedule of nirsevimab administration and the 5-month duration of nirsevimab efficacy, protection persisted during RSV inter-epidemic seasons, with a reduction of $17.5 \%(\sim 7300)$ RSV-MALRTIs among infants $<6$ months.

\section{Scenario 2: Assuming Reduction of RSV Viral Shedding by $50 \%$}

In scenario 2, a reduction in RSV-MALRTIs was predicted across age groups during the RSV epidemic and inter-epidemic seasons. This resulted from a direct effect on the nirsevimabeligible population and an indirect effect on the overall population, including nirsevimab-eligible individuals for whom nirsevimab was not effective (Fig. 3C; Table 2B). During the RSV epidemic season, the average RSV-MALRTI reduction was $51.9 \%(\sim 61,000)$ and $36.8 \%$ ( 40,000) RSV-MALRTIs for infants aged 0-6 months and 6-12 months, respectively. RSV-
MALRTI reduction among other age groups ranged from $2.0 \%$ for infants aged 12-24 months to $6.4 \%$ for those $>65$ years (Table 2B).

During the RSV inter-epidemic period, the average reduction in RSV-MALRTIs was $22.7 \%$ $(\sim 9,500)$ and $5.5 \%(\sim 2000)$ among infants aged 0-6 months and 6-12 months, respectively. Among other age groups, the average reduction ranged from $4.1 \%$ for children aged $12-24$ months to $8.3 \%$ for those $>65$ years (Table 2B).

Across age groups, indirect effects of nirsevimab during RSV seasons represented 15.5\% ( $\sim$ 19,000 RSV-MALRTIs) of all avoided cases in scenario 2 : $10.4 \% \quad(\sim 11,000 \quad$ RSV-MALRTIs $)$ during RSV epidemic seasons and 51.7\% ( 7800 RSV-MALRTIs) during RSV inter-epidemic seasons (Supplementary Material, Tables S3 and S4).

The model also predicted a different monthly distribution of RSV-MALRTI among infants aged $<24$ months, with episodes of RSVMALRTI observed early in the RSV season

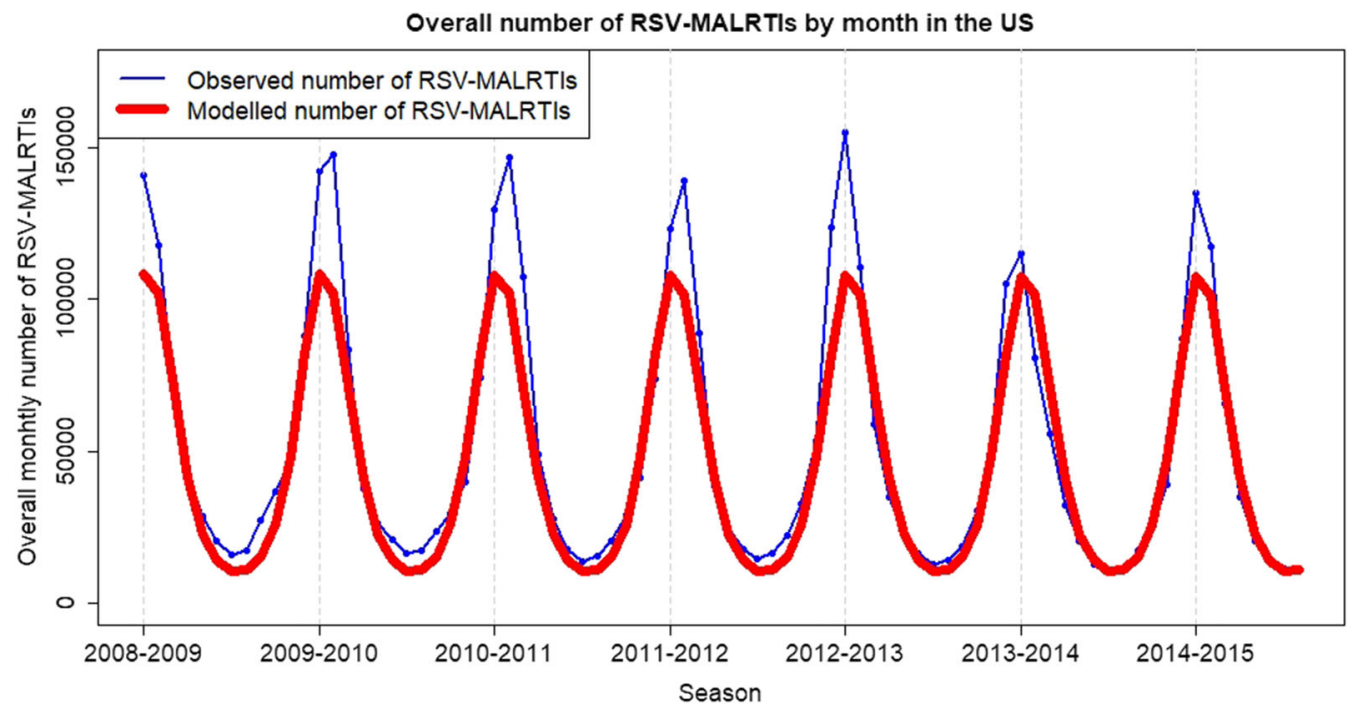

Fig. 2 Observed and modeled number of RSV-MALRTIs by season in the USA. Observed data (blue line) were based on MALRTIs reported between 1 January 2008 and 31 December 2015, and were obtained from the Truven Health Marketscan ${ }^{\circledR}$ Commercial Claims and Encounters, and Medicare Supplemental and Coordination of Benefits databases (Truven Health Analytics, Michigan, USA). The numbers of MALRTI due to RSV were calculated for the general US population the age-stratified US Census population estimates and projections from 2008 to 2015. Data from a deterministic age-structured compartmental model were fitted to the incidence of age-specific MALRTI by calendar month (red line) 
A

RSV-MALRTIS number in model with no nirsevimab

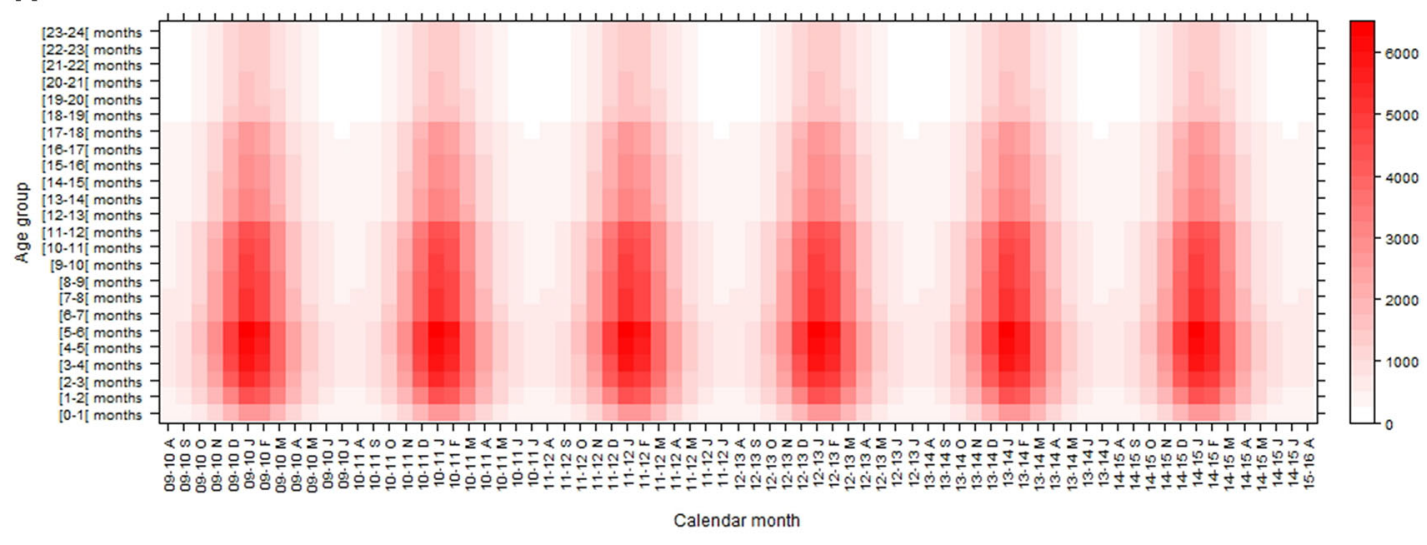

B
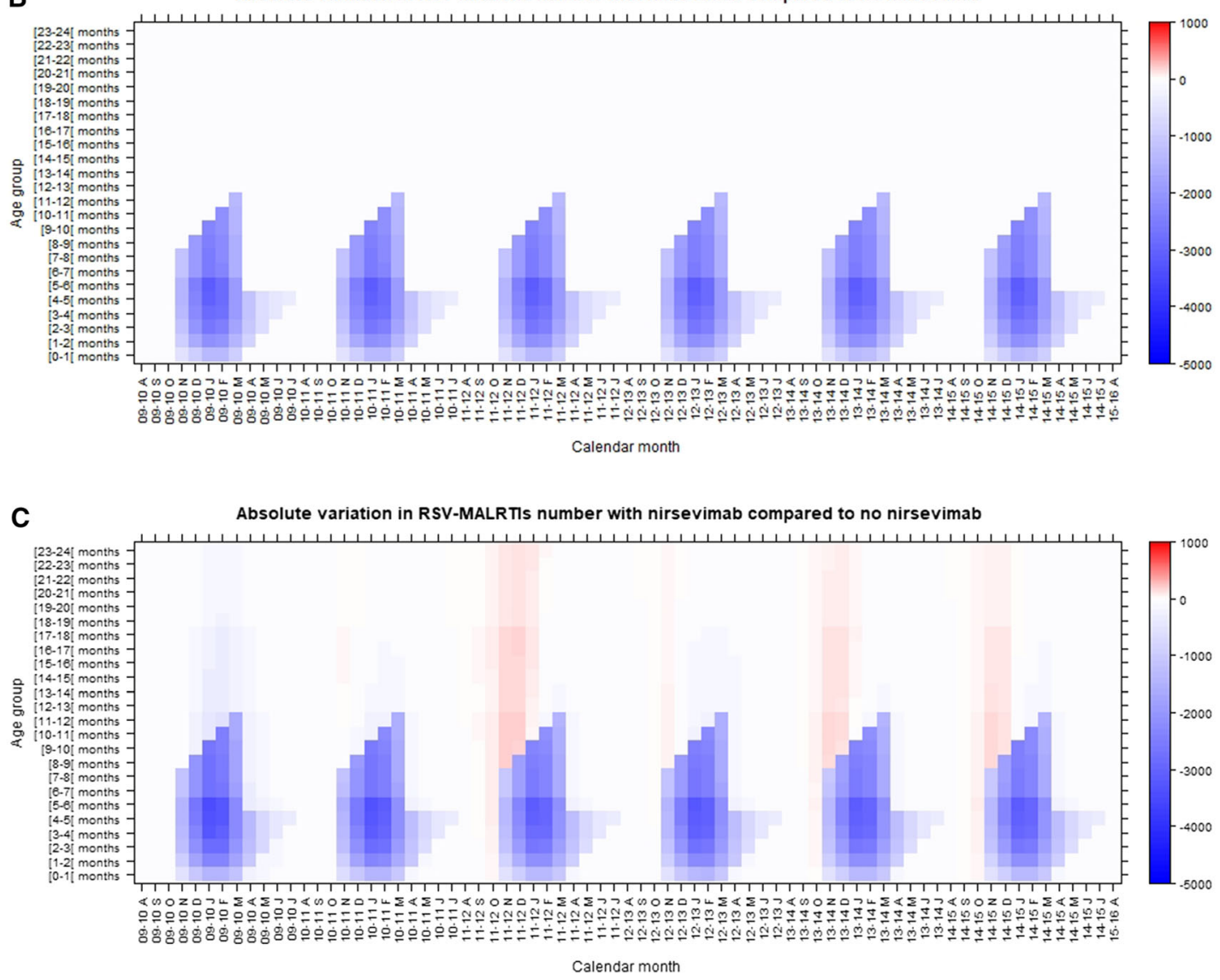

Fig. 3 Lexis diagrams showing the expected occurrence of RSV-MALRTIs by age group up to 24 months and by season, with no intervention (A), and the impact of nirsevimab administration given the assumptions of scenario 1 (no effect on RSV transmission (B), and scenario $2(50 \%$ reduction in viral shedding $(\mathbf{C})$. The graphs depict the number of RSV-MALRTIs for age cohorts up to 24 months (age, left $y$-axis), followed over time (calendar month, $x$-axis). In $\mathbf{B}$ and $\mathbf{C}$, blue denotes an absolute reduction in the number of RSV-MALRTIs, relative to the base scenario, in $\mathbf{A}$, red denotes an increase in numbers of RSV-MALRTI relative to the base scenario. The size of the reductions or increase in number of MALRTIs are reflected by the tone of color (right $y$-axis) 
(September/October), and an increase in the age at first infection (Fig. 3C). This shift in age for the first infection mainly affected infants aged 12-24 months of age (Supplementary Material, Fig. S7).

\section{Sensitivity Analyses}

The model without nirsevimab was most sensitive to the contact transmission probability, $\beta_{0}$; short-term protection and maternal antibody protection had very limited effect on model outputs (Supplementary Material, Fig. S8).

A possible overdiagnosis of RSV during the RSV inter-epidemic period was accounted for by a five-fold correction (i.e., reduction) of RSVMALRTI incidence between April and October each RSV season. The correction did not change the overall observed impact (Supplementary Material, Fig. S9).

Contact matrix sensitivity analysis provided similar results and did not change the potential impact of nirsevimab (Supplementary Material, Table S5).

Further sensitivity analyses per age group were performed to analyze the extent that the disease burden shifted to different age groups. The analysis of the effect of vaccine coverage, ranging from 100 to 0\%, (Supplementary Material, Fig. S10), and the multivariate sensitivity analyses on duration of maternal protective immunity, duration of short-term protection and transmission probability conducted in each age group, confirmed that the shift of disease was limited to the 12-24 month age group (Supplementary Material, Fig. S11).

\section{DISCUSSION}

Our findings suggest that, compared to no intervention, single-dose nirsevimab administered to all infants entering or born during their first RSV season may confer a significant benefit. Using this universal immunization strategy, based on these preliminary data, we can expect a reduction of MALRTIs among infants aged 0-6 months during the RSV epidemic season (November-March; scenario 1: $-50 \%$, scenario 2: $-51.9 \%)$ and during RSV inter-epidemic seasons (scenario $1:-18 \%$, scenario
$2:-22.7 \%)$, and among infants aged 6-12 months during the RSV epidemic season (scenario $1:-35 \%$, scenario $2:-36.8 \%$ ). The smaller reduction during the RSV season in this age group is explained by the fact that only a proportion of infants born within the season (from December to March) receive nirsevimab at birth (during their first RSV season), benefiting from its protection. However, they experience a second RSV season prior to their first year of age, when no prevention is planned. Results from scenario 1, comparable to a static model, are consistent with previous findings (ranging between 48.2 and $54.7 \%$ depending on the type of health care resource utilizations) [32]. Our slightly lower estimates are due to different assumptions on efficacy.

Considering scenario 2, based on the assumption that viral shedding is reduced by $50 \%$, with a consequent reduction in transmission, herd protection can be expected across age groups during the RSV epidemic and inter-epidemic seasons. This indirect effect of nirsevimab could account for $16 \%$ of all avoided cases. Additionally, the onset of an epidemic among infants aged $<24$ months could be expected to shift from October/November to September/October, albeit with an expected reduction in the number of MALRTIs overall compared to no intervention. Moreover, an increase in the age at first infection during the next RSV epidemic season could be expected, with a shift mainly towards the 12-24 month age group between September and October. Multivariate sensitivity analyses on the duration of maternal protective immunity, duration of short-term protection, and transmission probability in each age group, as well as sensitivity analysis on coverage rate, confirmed that this effect was limited to the 12-24 month age group, with a few additional cases each season. These two effects result from two well-known epidemiological mechanisms. Firstly, due to the reduced force of infection during the first RSV season, a pool of infants remain fully susceptible and experience their first exposure to RSV during their second season; however, such children would be older and thus would present improved lung maturity and be less likely to develop a severe event. Secondly, this 


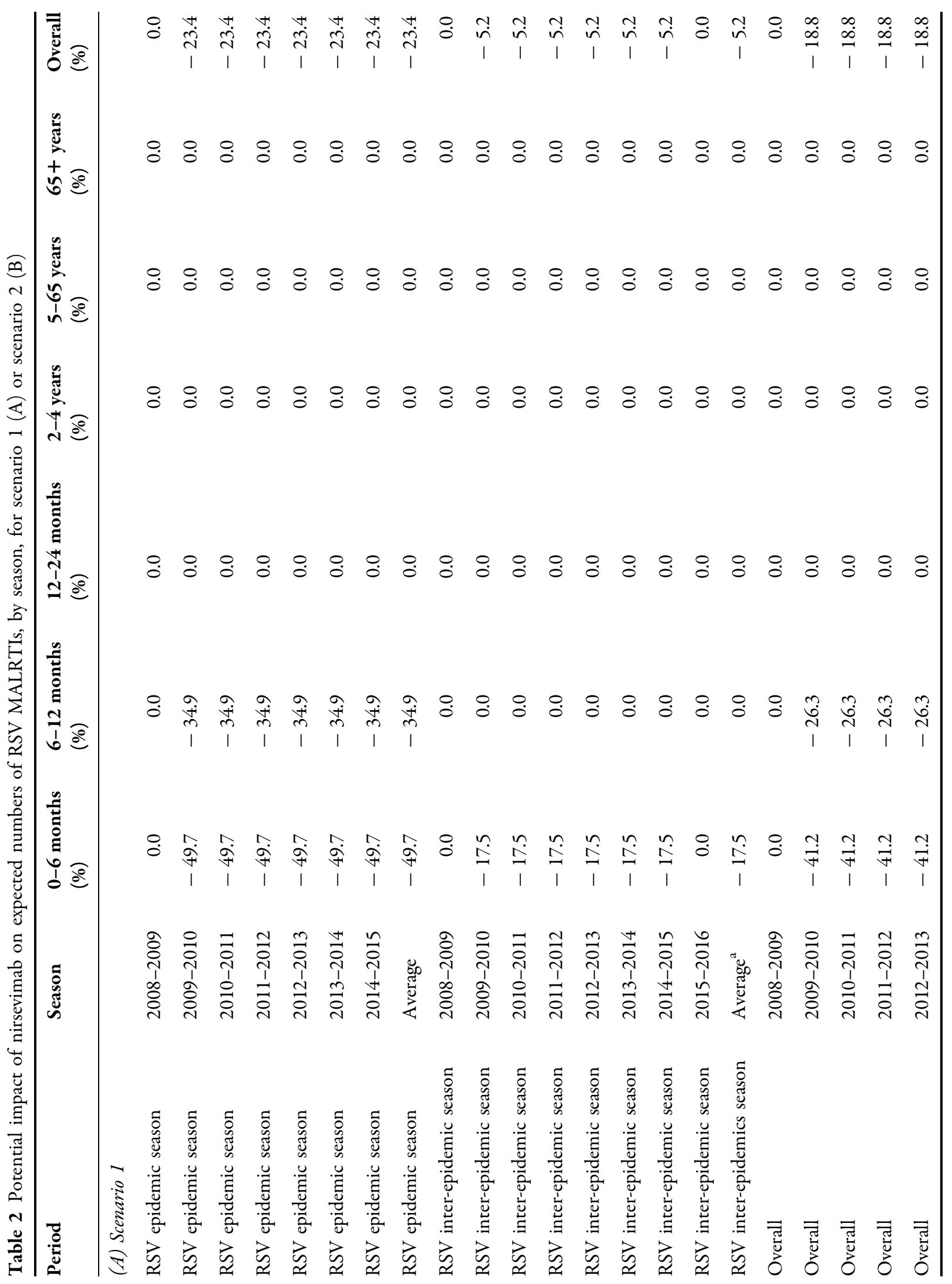




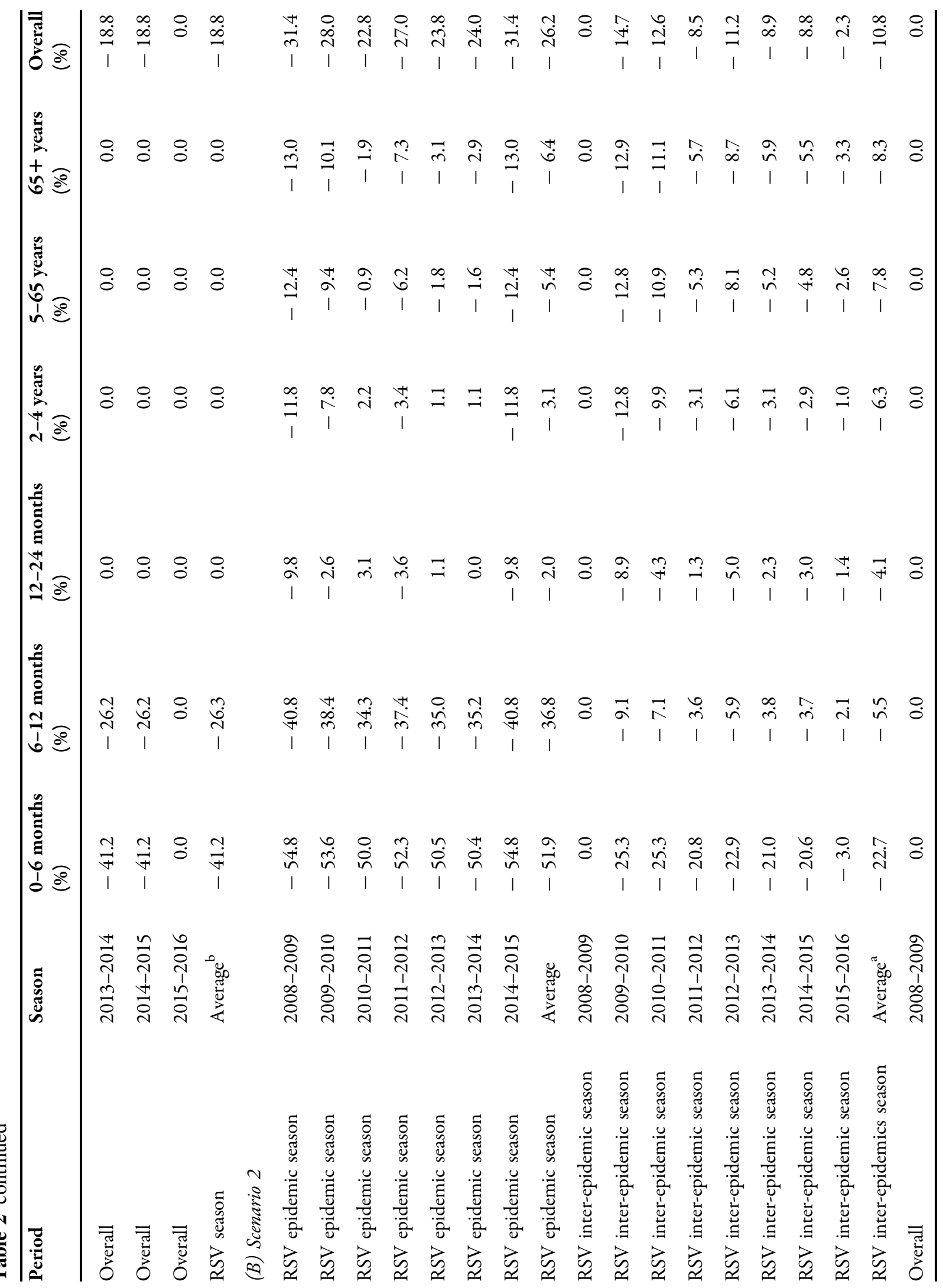




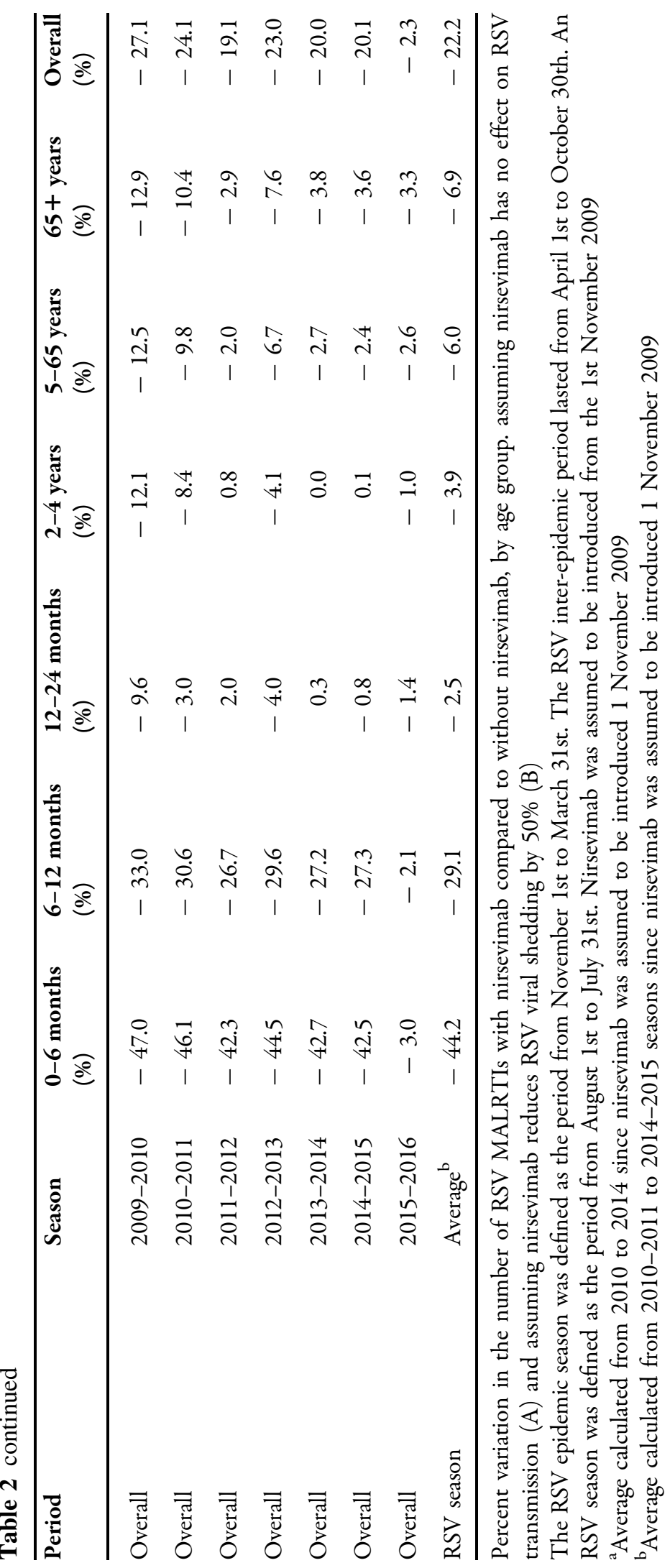


additional pool of susceptible infants becoming infected at the beginning of the second season results in increased exposure of younger age groups, explaining the earlier onset in September/October in infants aged $<24$ months. Despite this shift and increase of cases in the second RSV season, the balance of indirect effect remains positive, with a higher level of reduction overall.

These results rely on several assumptions related to modeling and data. Regarding RSV infection and disease, our model structure is similar to other deterministic age-structured compartmental models aimed at reflecting RSV natural history $[19,20]$, particularly with regards to the successive phases of susceptibility (S0, S1, S2) depicting immature immune systems in younger infants (aged $<12$ months) who are more affected by severe and fatal outcomes [36]. One strength of our model was a more detailed analysis by age group than previous models by incorporating 32 age classes that covered the whole population, including an unprecedented level of granularity for those $<24$ months. Also, consistent with previous reports fitting models to RSV epidemiological data, we successfully captured seasonal patterns of RSV epidemics and disease burden for each age group, with a good level of agreement between model outputs and observed data $[19,20,30]$. As in other models, variability of the peak amplitude of seasonal epidemics was not captured as well. This may be, at least partly, due to the fact that our model is not built on viral group structure and thus does not take into account potential differences between the circulating viral groups (A or B) in transmission dynamics and the susceptibility of individuals [37]. As we considered averages over multiple seasons, this is unlikely to affect the interpretation of our data in relation to the objective of the study; this does, however, remain an important factor to be addressed in future work.

Uncertainties around specific parameters led us to conduct sensitivity analyses. The uncertainty around maternal protection and shortterm protection did not affect RSV transmission dynamics. Probability of transmission sensitivity analysis showed, not surprisingly, that this is a main driver of amplitude of seasonal RSV epidemics, validating our choice to estimate it during the calibration process. We used a previously published synthetic contact matrix [38], to our knowledge the only available material for the USA that could be used in our model. Data on contact structures for children $<5$ years old are lacking, and contact matrices have not been estimated at the same level of granularity as used in our model. We therefore extended and rearranged the published USA contact matrix to fit our model age structure, assuming homogeneity of contacts within age groups $<5$ years. We nevertheless assessed whether an increase in contact rates within age groups up to 5 years would affect the results, and concluded similarly about the potential impact of nirsevimab. However, since most contacts occur in persons $>5$ years old, the contact matrix used may underlie the small size of the effects observed in the current analysis. Further knowledge on contact structures at young ages would enable such a contact matrix to be adapted more precisely to our model. Finally, the 50\% reduction of transmission was arbitrarily chosen to exemplify the potential impact of the effect of nirsevimab on transmission. Additional data from clinical trials, translational research, and real-world evidence will help to refine this figure and improve understanding of the broader value of nirsevimab.

Regarding the data used as input, under base scenario assumptions (no intervention), our model estimated, consistently with observed data, incidence rates of RSV-MALRTIs of 0.8-1.3 per 1000 persons per season for the whole population, with the highest burden of RSVMALRTIs occurring in infants aged $<12$ months. The burden remained significant up to 5 years of age, with a decreasing trend as children grow older. The lowest rate was observed in the age group 5-65 years, with RSV-MALRTI incidence appearing to increase again after 65 years of age. It should be noted that the data were limited to working-age individuals and their families with commercial health coverage or private Medicare Supplement coverage, and excluded those who are uninsured or affiliated to Medicaid. Therefore, these data do not necessarily accurately reflect 
Table 3 Comparison of RSV hospitalization rates (per 1000)

\begin{tabular}{|c|c|c|c|c|c|c|c|}
\hline & \multicolumn{4}{|c|}{ Prospective studies } & \multicolumn{3}{|c|}{ Claims database } \\
\hline & $\begin{array}{l}\text { Study } \\
\text { finding }\end{array}$ & $\begin{array}{l}\text { Rha } \\
(2020) \\
{[42]}\end{array}$ & $\begin{array}{l}\text { Arriola } \\
(2019)[43]\end{array}$ & $\begin{array}{l}\text { Hall } \\
(2013) \\
{[9]}\end{array}$ & $\begin{array}{l}\text { McLaurin } \\
\text { Medicaid } \\
(2016) \\
{[44]}\end{array}$ & $\begin{array}{l}\text { McLaurin } \\
\text { Commercial (2016) } \\
{[44]}\end{array}$ & $\begin{array}{l}\text { Stockman } \\
(2012)\end{array}$ \\
\hline $0-11$ months & 7.46 & $9.75^{\mathrm{a}}$ & $9.98^{a}$ & $8.84^{\mathrm{a}}$ & 17.8 & 9.98 & 26.0 \\
\hline
\end{tabular}

${ }^{\mathrm{a} C a l c u l a t e d}$ based on rates given per age groups

the general population. Furthermore, the ICD9-CM codes used to identify RSV cases may be wrongly or not assigned in claims records, leading to a probable underestimation of the burden of MALRTI. Compared to existing literature, our figures seem to underestimate the burden of RSV-MALRTIs [39]. Indeed, the case definition for RSV-MALRTIs here differs from the definition used in other epidemiological studies, but was chosen to fit with the clinical primary endpoints studied in the Phase $2 b$ [14] and Phase 3 [15] trials assessing nirsevimab. This approach may be considered conservative, but it remains internally valid as our objective was to understand the impact of nirsevimab with universal use, considering the uncertainties surrounding the mechanism of action. A recent study modeling the burden of RSVMALRTIs in the USA found 587,780 cases in infants aged $0-1$ year [32]. We estimated a seasonal average of 299,672 RSV-MALRTIs in this birth cohort, a potential underestimation of almost 50\%. This limitation may be linked to factors independent of the RSV transmission dynamics, and thus would not necessarily affect our estimation of the positive impact of nirsevimab. Additionally, we were concerned by an unexpected observed occurrence of RSV-MALRTIs between epidemic seasons. This may be explained by varying incidence patterns between different states (e.g., a longer RSV epidemic season in Florida, compared to other states) [40] or by inappropriate coding of respiratory symptoms as RSV episodes in the MarketScan databases. We assessed whether a possible overdiagnosis of RSV during RSV interepidemic periods modified the results, but no changes were observed on the impact of nirsevimab. Finally, we observed an apparent overdiagnosis for the 2-4 years age group, potentially due to an imbalance in the assignment of ICD codes. However, the model gave similar results overall, regardless of whether this age group was considered during the calibration process.

Given these limitations, we attempted to validate our approach using RSV hospitalizations, which are considered to be exclusively related to lower respiratory tract infection [32], thus facilitating comparisons with other sources. In a MarketScan research analysis in the USA from 2008 to 2014, hospitalizations represented $9.9 \%$ of RSV-related healthcare resource utilization among infants aged $<12$ months. This value was $7.2 \%$ in the whole population [41]. Applying these figures to our data gives an estimated hospitalization rate in our study of 7.46 per 1000 infants. Table 3 shows a comparison between this and published rates of hospitalizations in infants in the USA. The RSV hospitalization rate in our model is consistent with those in published prospective studies, reinforcing our approach. This is expected since hospital utilization most likely leads to a more accurate RSV case ascertainment, as it is generally confirmed by virologic testing.

\section{CONCLUSIONS}

These preliminary investigations provide a deeper insight into the potential benefits of administering single-dose nirsevimab in infants during their first RSV season. Our model provides an in-depth analysis of RSV-associated MALRTI claims data by age group, with a particularly high level of granularity over the first 24 months of life. Such observations could help 
to inform authorities on the appropriate timing of nirsevimab regimens. Our data, which were based on different assumptions about the mechanism of action of nirsevimab, predicted a shift in susceptibility to RSV-MALRTIs towards older age groups among children. Nevertheless, the predicted net impact on the occurrence of seasonal RSV-MALRTIs remained positive, both overall and when analyzed by age group. The successful implementation of a nirsevimab regimen will depend on the actual coverage achieved, the duration of nirsevimab protection, and the potential effects of RSV antibodies on the infectiousness of the virus and the susceptibility of infants and children to infection. These uncertainties will be addressed through phase 3 clinical trials and future analysis of realworld data following potential licensure of nirsevimab. Our model may be a useful tool to perform sensitivity analyses, further analyze implementation strategies for RSV illness prophylaxis in the future, and assess the economic impact of these strategies.

\section{ACKNOWLEDGEMENTS}

Funding. The study was jointly funded by Sanofi Pasteur and AstraZeneca. Sanofi Pasteur funded the journal's Rapid Service Fee.

Medical Writing, Editorial, and Other Assistance. Medical writing assistance was provided by Juliette Gray, PhD, of inScience Communications, Springer Healthcare Ltd, UK, which was funded by Sanofi Pasteur. For coordination of manuscript development, the authors would like to thank Isabel Gregoire, $\mathrm{PhD}$, at Sanofi Pasteur. Editorial support was provided by Anirban Sanyal, PhD, at Sanofi.

Authorship. All named authors meet the International Committee of Medical Journal Editors (ICMJE) criteria for authorship for this article, take responsibility for the integrity of the work as a whole, and have given their approval for this version to be published.
Author Contributions. All authors were involved in the conception and design of the study, and in the methodology, analysis and interpretation of the data. Clarisse Demont and Alexia Kieffer obtained access to the data. Nicolas Voirin and Victor Virlogeux wrote the original manuscript; all authors were involved in revising subsequent versions and approving the final version for submission.

Disclosures. Clarisse Demont and Alexia Kieffer are employees of Sanofi Pasteur and hold Sanofi shares. Nicolas Voirin holds shares of EPIMOD which is a subcontractor of Sanofi, and Victor Virlogeux is a consultant for EPIMOD.

Compliance with Ethics Guidelines. All database records were de-identified in compliance with US patient confidentiality requirements, including the Health Insurance Portability and Accountability Act of 1996. Institutional Review Board approval was not required as we used only de-identified patient records (see Supplementary Methods).

Data Availability. The program code and datasets generated and/or analyzed during the current study are available from the corresponding author on reasonable request.

Open Access. This article is licensed under a Creative Commons Attribution-NonCommercial 4.0 International License, which permits any non-commercial use, sharing, adaptation, distribution and reproduction in any medium or format, as long as you give appropriate credit to the original author(s) and the source, provide a link to the Creative Commons licence, and indicate if changes were made. The images or other third party material in this article are included in the article's Creative Commons licence, unless indicated otherwise in a credit line to the material. If material is not included in the article's Creative Commons licence and your intended use is not permitted by statutory regulation or exceeds the permitted use, you will need to obtain permission directly from the copyright holder. To view a copy of this licence, visit http://creativecommons.org/licenses/by$\mathrm{nc} / 4.0 /$. 


\section{REFERENCES}

1. Shi T, McAllister DA, O'Brien KL, et al. Global, regional, and national disease burden estimates of acute lower respiratory infections due to respiratory syncytial virus in young children in 2015: a systematic review and modelling study. Lancet. 2017;390(10098):946-58.

2. Broberg EK, Waris M, Johansen K, Snacken R, Penttinen P, European Influenza Surveillance Network. Seasonality and geographical spread of respiratory syncytial virus epidemics in 15 European countries, 2010 to 2016. Euro Surveill. 2018;23(5): 17-00284.

3. Rose EB, Wheatley A, Langley G, Gerber S, Haynes A. Respiratory syncytial virus seasonality-United States, 2014-2017. Morb Mortal Wkly Rep. 2018;67(2):71-6.

4. Glezen WP, Taber LH, Frank AL, Kasel JA. Risk of primary infection and reinfection with respiratory syncytial virus. Am J Dis Child. 1986;140(6):543-6.

5. Stockman LJ, Curns AT, Anderson LJ, Fischer-Langley G. Respiratory syncytial virus-associated hospitalizations among infants and young children in the United States, 1997-2006. Pediatr Infect Dis J. 2012;31(1):5-9.

6. Tong S, Amand C, Kieffer A, Kyaw M. Incidence of respiratory syncytial virus related healthcare utilization in the United States. Value Health. 2017;20(9):A781.

7. Zhou H, Thompson WW, Viboud CG, et al. Hospitalizations associated with influenza and respiratory syncytial virus in the United States, 1993-2008. Clin Infect Dis. 2012;54(10):1427-36.

8. Langley GF, Anderson LJ. Epidemiology and prevention of respiratory syncytial virus infections among infants and young children. Pediatr Infect Dis J. 2011;30(6):510-7.

9. Hall CB, Weinberg GA, Blumkin AK, et al. Respiratory syncytial virus-associated hospitalizations among children less than 24 months of age. Pediatrics. 2013;132(2):e341-8.

10. Committee on infectious diseases and bronchiolitis guidelines committee. From the American Academy of Pediatrics. Updated guidance for palivizumab prophylaxis among infants and young children at increased risk of hospitalization for respiratory syncytial virus infection. Pediatrics. 2014;134(2):415-20.

11. Pavilack M, Clifford RA, Gonzales T, Kong AM, Wade S, McLaurin KK. Trends in utilization of outpatient respiratory syncytial virus prophylaxis with palivizumab among medicaid- and commercially insured infants. Infect Dis Ther. 2018;7(1): 121-34.

12. PATH. RSV Vaccine and mAb Snapshot. Vaccine Resource Library. 2019. https://vaccineresources. org/details.php?i=1562. Accessed 17 Sep 2019.

13. Domachowske JB, Khan AA, Esser MT, et al. Safety, tolerability, and pharmacokinetics of MEDI8897, an extended half-life single-dose respiratory syncytial virus prefusion F-targeting monoclonal antibody administered as a single dose to healthy preterm infants. Pediatr Infect Dis J. 2018;2018:1.

14. Griffin MP, Yuan Y, Takas T, et al. Single-dose nirsevimab for prevention of RSV in preterm infants. N Engl J Med. 2020;383(5):415-25.

15. ClinicalTrials.gov [NCT03979313]. A Study to Evaluate the Safety and Efficacy of MEDI8897 for the Prevention of Medically Attended RSV LRTI in Healthy Late Preterm and Term Infants (MELODY) [unpublished]. 2021. https://clinicaltrials.gov/ct2/ show/ NCT03979313. Accessed 21 Jan 2021.

16. Sanof Press Release, 26 April 2021. 2021. https:// www.sanofi.com/en/media-room/press-releases/ 2021/2021-04-26-08-00-00-2216474. Accessed 01 Oct 2021.

17. Cromer D, van Hoek AJ, Newall AT, Pollard AJ, Jit M. Burden of paediatric respiratory syncytial virus disease and potential effect of different immunisation strategies: a modelling and cost-effectiveness analysis for England. Lancet Public Health. 2017;2(8):e367-74.

18. Li X, Willem L, Antillon M, Bilcke J, Jit M, Beutels P. Health and economic burden of respiratory syncytial virus (RSV) disease and the cost-effectiveness of potential interventions against RSV among children under 5 years in 72 Gavi-eligible countries. BMC Med. 2020;18(1):82.

19. Kinyanjui TM, House TA, Kiti MC, Cane PA, Nokes DJ, Medley GF. Vaccine induced herd immunity for control of respiratory syncytial virus disease in a low-income country setting. PLoS ONE. 2015;10(9): e0138018.

20. Pan-Ngum W, Kinyanjui T, Kiti M, et al. Predicting the relative impacts of maternal and neonatal respiratory syncytial virus (RSV) vaccine target product profiles: a consensus modelling approach. Vaccine. 2017;35(2):403-9.

21. Chu HY, Steinhoff MC, Magaret A, et al. Respiratory syncytial virus transplacental antibody transfer and kinetics in mother-infant pairs in Bangladesh. J Infect Dis. 2014;210(10):1582-9. 
22. Nyiro JU, Sande C, Mutunga M, et al. Quantifying maternally derived respiratory syncytial virus specific neutralising antibodies in a birth cohort from coastal Kenya. Vaccine. 2015;33(15): 1797-801.

23. Hall CB, Walsh EE, Long CE, Schnabel KC. Immunity to and frequency of reinfection with respiratory syncytial virus. J Infect Dis. 1991;163(4):693-8.

24. Okiro EA, White LJ, Ngama M, Cane PA, Medley GF, Nokes DJ. Duration of shedding of respiratory syncytial virus in a community study of Kenyan children. BMC Infect Dis. 2010;10:15.

25. Agoti CN, Mwihuri AG, Sande CJ, et al. Genetic relatedness of infecting and reinfecting respiratory syncytial virus strains identified in a birth cohort from rural Kenya. J Infect Dis. 2012;206(10): 1532-41.

26. Ohuma EO, Okiro EA, Ochola R, et al. The natural history of respiratory syncytial virus in a birth cohort: the influence of age and previous infection on reinfection and disease. Am J Epidemiol. 2012;176(9):794-802.

27. Hall CB, Geiman JM, Biggar R, Kotok DI, Hogan PM, Douglas GR. Respiratory syncytial virus infections within families. N Engl J Med. 1976;294(8):414-9.

28. Henderson FW, Collier AM, Clyde WA, Denny FW. Respiratory-syncytial-virus infections, reinfections and immunity. A prospective, longitudinal study in young children. N Engl J Med. 1979;300(10):530-4.

29. Monto AS, Bryan ER, Rhodes LM. The Tecumseh study of respiratory illness. VII. Further observations on the occurrence of respiratory syncytial virus and Mycoplasma pneumoniae infections. Am J Epidemiol. 1974;100(6):458-68.

30. Pitzer VE, Viboud C, Alonso WJ, et al. Environmental drivers of the spatiotemporal dynamics of respiratory syncytial virus in the United States. PLoS Pathog. 2015;11(1):e1004591.

31. Nokes DJ, Okiro EA, Ngama M, et al. Respiratory syncytial virus infection and disease in infants and young children observed from birth in Kilifi District, Kenya. Clin Infect Dis. 2008;46(1):50-7.

32. Rainisch G, Adhikari B, Meltzer MI, Langley G. Estimating the impact of multiple immunization products on medically-attended respiratory syncytial virus (RSV) infections in infants. Vaccine. 2020;38(2):251-7.
33. Griffin P. Ph2b Outcomes of the MedImmune MEDI8897 RSV mAb. RSVVW19. 2019.

34. Zhu Q, McLellan JS, Kallewaard NL, et al. A highly potent extended half-life antibody as a potential RSV vaccine surrogate for all infants. Sci Transl Med. 2017;9(388):1928.

35. Truven health analytics. Comparative effectiveness research. 2012. https://truvenhealth.com/portals/0/ assets/ACRS_11228_0912_CompEffectResearch_SS_ Web.pdf. Accessed 17 Sep 2019.

36. Openshaw PJM, Chiu C, Culley FJ, Johansson C. Protective and harmful immunity to RSV infection. Annu Rev Immunol. 2017;35:501-32.

37. White LJ, Waris M, Cane PA, Nokes DJ, Medley GF. The transmission dynamics of groups $A$ and $B$ human respiratory syncytial virus (hRSV) in England \& Wales and Finland: seasonality and crossprotection. Epidemiol Infect. 2005;133(2):279-89.

38. Prem K, Cook AR, Jit M. Projecting social contact matrices in 152 countries using contact surveys and demographic data. PLoS Comput Biol. 2017;13:9.

39. Cai W, Tolksdorf K, Hirve S, et al. Evaluation of using ICD-10 code data for respiratory syncytial virus surveillance. Influenza Other Respir Viruses. 2020;14(6):630-7.

40. McGuiness CB, Boron ML, Saunders B, Edelman L, Kumar VR, Rabon-Stith KM. Respiratory syncytial virus surveillance in the United States, 2007-2012: results from a national surveillance system. Pediatr Infect Dis J. 2014;33(6):589-94.

41. Tong S, Amand C, Kieffer A, Kyaw MH. Incidence of respiratory syncytial virus related health care utilization in the United States. J Glob Health. 2020;10(2):020422.

42. Rha B, Curns AT, Lively JY, et al. Respiratory syncytial virus-associated hospitalizations among young children: 2015-2016. Pediatrics. 2020;146:1.

43. Arriola CS, Kim L, Langley G, et al. Estimated burden of community-onset respiratory syncytial virusassociated hospitalizations among children aged $<2$ years in the United States, 2014-15. J Pediatric Infect Dis Soc. 2020;9(5):587-95.

44. McLaurin KK, Farr AM, Wade SW, Diakun DR, Stewart DL. Respiratory syncytial virus hospitalization outcomes and costs of full-term and preterm infants. J Perinatol. 2016;36(11):990-6. 